\title{
Effect of Heart Rate and Body Position on the Complexity of the QRS and T Wave in Healthy Subjects
}

\author{
VN Batchvarov ${ }^{1}$, G Bortolan$^{2}$, II Christov ${ }^{3}$ \\ ${ }^{1}$ St George's University of London, London, United Kingdom \\ ${ }^{2}$ Institute of Biomedical Engineering ISIB - CNR, Padova, Italy \\ ${ }^{3}$ Centre of Biomedical Engineering, Bulgarian Academy of Sciences, Sofia, Bulgaria
}

\begin{abstract}
We analysed the effect of heart rate and body position on the complexity of the QRS and T wave quantified by the ratio of $2^{\text {nd }} / 1^{\text {st }}$ eigenvalue from principal component analysis (PCA) (QRS-PCA, T-PCA) using continuous 25 min 12-lead digital ECGs (500 Hz, $4.88 \mu \mathrm{V}$ resolution) acquired on 2 occasions in supine and standing position in 15 healthy subjects ( 8 men, age $28.6 \pm 7.5$ years). In the group as a whole, QRS-PCA and T-PCA did not differ significantly between the two positions. However, the linear correlation coefficient between the PCA parameters and the RR interval varied widely between different subjects in the supine position (QRS-PCA: from 0.002 to 0.61 ; T-PCA: from 0.01 to 0.65 ) and even more in standing position (QRS-PCA: from -0.55 to 0.48 ; $T$ PCA: from -0.63 to 0.51). In both positions, the intrasubject variability of QRS-PCA and T-PCA was significantly smaller than the inter-subject variability.
\end{abstract}

\section{Introduction}

Principal component analysis (PCA) quantifies the complexity of the ECG waves by defining a set of independent forms (components) with decreasing relative value, which can fully describe their shape. Visibly more complex QRS or T waves with irregularities, notches, etc., which are frequently observed in diseased hearts are described by a greater number of independent components, with increasing relative value of the smaller components.

Whilst the diagnostic [1 $\underline{-} \underline{3}]$ and prognostic $[\underline{4}, \underline{5}]$ value of PCA of the T wave has been demonstrated, the effect of physiological factors on the QRS and $T$ wave complexity is unknown. The $\mathrm{T}$ wave shape or polarity can be influenced by age, sex, heart rate, body position, autonomic activity, respiration, temperature, electrolyte concentration, food and mental activity [ㅁ-10]. It is possible that these factors can also affect $T$ wave complexity. We investigated the effect of heart rate and body position on QRS and T wave complexity using an ECG database recorded in healthy subjects.

\section{Methods}

A digital ECG database was previously recoded for another project in 15 subjects ( 8 men, 7 women, age $28.6 \pm 7.5$ years, range 21.5 - 49.1 years) with negative medical history, normal physical examination and normal resting 12-lead ECG, who were not taking any medications during the project.

Data were acquired after 5 minutes of rest in the supine position. In each subject, a continuous digital 12lead ECG was recorded for 25 minutes (5 minutes supine $\rightarrow 10$ minutes standing $\rightarrow 10$ minutes supine) with Mason-Likar electrode positions [11] using a PC-based 12-lead ECG recorder (CardioSoft ${ }^{\mathrm{TM}}$, GE Medical Systems, Milwaukee, USA, $500 \mathrm{~Hz}, 4.88 \mu \mathrm{V}$ resolution). In each individual, the same protocol was repeated after approximately 1 week at the same time of the day (within \pm 1 hour). The local Ethics Committee approved the study protocol and each subject provided a written consent.

\subsection{Data analysis}

All ECG recordings were visually assessed on screen using the CardioSoft ${ }^{\mathrm{TM}}$ software programme in order to exclude segments with noise or premature beats. The first 2 minutes and the last 30 seconds of recording in each position were excluded from the study, and 2.5 minutes supine $\rightarrow 7.5$ minutes standing $\rightarrow 7.5$ minutes supine were analysed. The ECG were preprocessed suppressing power-line interference, electromyogram noise and baseline drift according to our previously published material on Q-onset and T-end delineation [12].

QRS detection was performed following the work of Christov [13], then one complex lead was synthesised from the initials 8 leads [14]. The QRS and the T wave boundaries as well as the RR interval were delineated and 
measured on the complex lead [12].

All ECG recordings and the delineated boundaries were visually observed, and corrected if necessary. Preliminary ventricular contractions and noisy heart beats were manually excluded from the results.

The PCA analysis was performed on the intervals of QRS and T waves, considering all 12 ECG leads. The ratio of the $2^{\text {nd }}$ to $1^{\text {st }}$ Eigenvalue was taken for analysis (QRS-PCA and T-PCA, respectively).

\subsection{Statistical analysis}

Data were compared using Wilcoxon non-parametric test. The relation of PCA-QRS and PCA-T with heart rate was assessed with Pearson correlation coefficient. In order to compare the intra-subject with the inter-subject variability, for each parameter the absolute differences between the values of the $1^{\text {st }}$ and $2^{\text {nd }}$ recordings in each subject were compared with the absolute differences between the average values from the 2 recordings in any 2 subject (105 possible combinations) using Wilcoxon test. Data are presented as median (range) unless stated otherwise. Statistically significant difference was accepted as $\mathrm{p}<0.05$.

\section{Results}

The period between the 2 recordings was 7 (4-8) days. The first 2 minute and 30 seconds and the second $7 \mathrm{~min}$ and 30 seconds recording in the supine position were analysed together, making $10 \mathrm{~min}$ in the supine and $7 \mathrm{~min}$ and 30 seconds in standing position in all subjects. The total number of analysed PCA-QRS and PCA-T for the 15 individuals was 33165 (16580 in supine and 16585 in standing position).

As expected, the mean RR interval was significantly shorter in standing compared to supine position in both recordings $(767 \mathrm{~ms}(634,1263)$ vs $967 \mathrm{~ms}(831,1438)$, $\mathrm{P}<0.001$ and $790 \mathrm{~ms}(662,1132)$ vs $1031 \mathrm{~ms}(805,1474)$, $\mathrm{P}<0.001)$. There were no statistically significant differences in QRS-PCA $(0.404(0.055,0.663)$ vs 0.357 $(0.058,0.682), \mathrm{P}=0.82$ and $0.400(0.051,0.570)$ vs 0.426 $(0.054,0.734), \mathrm{P}=11)$ and T-PCA $(0.025(0.005,0.191)$ vs $0.025(0.001,0.222), \mathrm{P}=0.39$ and $0.024(0.006,0.154)$ vs $0.023(0.001,0.403), \mathrm{P}=0.43)$ between supine and standing position.

However, the correlation between the PCA parameters and $\mathrm{RR}$ interval in the supine position varied widely between individual subjects from practically no correlation to a moderate positive one, with no significant difference between the 2 recordings (QRS-PCA: 0.26 $(0.002-0.61)$ and $0.22(0.02-0.55)$ during the $1^{\text {st }}$ and $2^{\text {nd }}$ recording respectively, $\mathrm{P}=0.57$; T-PCA: $0.28(0.03-0.65)$ and $0.19(0.01-0.63)$, respectively, $\mathrm{P}=0.33)$. In standing position, the PCA/RR correlation varied even more, from moderate negative in some subjects to moderate positive in others (QRS-PCA: $0.15(-0.55-0.46)$ and $0.15(-0.40$ $0.48)$ for the $1^{\text {st }}$ and $2^{\text {nd }}$ recording, respectively, $\mathrm{P}=0.86$; T-PCA: 0.03 (-0.63- 0.51) and 0.02 (-0.54-0.47), $\mathrm{P}=0.73)$. The differences between supine and standing position were significant for T-PCA/RR $(\mathrm{P}=0.01$ and $\mathrm{P}=0.008$ for the $1^{\text {st }}$ and $2^{\text {nd }}$ recording, respectively) but not for $\mathrm{QRS}-\mathrm{PCA} / \mathrm{RR}(\mathrm{P}=0.23$ and $\mathrm{P}=0.07)$.

Table 1 Individual QRS-PCA/RR interval correlation coefficients

\begin{tabular}{ccccc}
\hline \hline & \multicolumn{2}{c}{$1^{\text {st }}$ recording } & \multicolumn{2}{c}{$2^{\text {nd }}$ recording } \\
\hline & Supine & Standing & Supine & Standing \\
\hline 1 & $0.41^{*}$ & $0.42^{*}$ & $0.32^{*}$ & $0.12^{*}$ \\
2 & $0.43^{*}$ & $-0.43^{*}$ & $0.13^{*}$ & $-0.25^{*}$ \\
3 & $0.61^{*}$ & $0.15^{*}$ & $0.39^{*}$ & $0.48^{*}$ \\
4 & $0.20^{*}$ & $-0.19^{*}$ & $0.55^{*}$ & $0.25^{*}$ \\
5 & $0.23^{*}$ & $0.13^{*}$ & $0.36^{*}$ & $0.14^{*}$ \\
6 & $0.26^{*}$ & 0.04 & $0.33^{*}$ & $0.21^{*}$ \\
7 & $0.28^{*}$ & $0.16^{*}$ & $0.37^{*}$ & $-0.17^{*}$ \\
8 & $0.44^{*}$ & $-0.55^{*}$ & 0.06 & $-0.40^{*}$ \\
9 & $0.35^{*}$ & $0.09^{\wedge}$ & 0.03 & 0.02 \\
10 & $0.12^{*}$ & $0.46^{*}$ & $0.20^{*}$ & $-0.28^{*}$ \\
11 & $0.12^{*}$ & $0.12^{*}$ & 0.04 & $0.09^{\wedge}$ \\
12 & $0.15^{*}$ & $0.15^{*}$ & $0.15^{*}$ & $0.20^{*}$ \\
13 & $0.37^{*}$ & $0.40^{*}$ & $0.31^{*}$ & $0.39^{*}$ \\
14 & 0.04 & $0.41^{*}$ & 0.02 & $0.17^{*}$ \\
15 & 0.00 & $0.29^{*}$ & $0.22^{*}$ & $0.26^{*}$ \\
\hline \hline
\end{tabular}

Table 2 Individual T-PCA/RR interval correlation coefficients

\begin{tabular}{ccccc}
\hline \hline & \multicolumn{2}{c}{$1^{\text {st }}$ recording } & \multicolumn{2}{c}{$2^{\text {nd }}$ recording } \\
\hline & Supine & Standing & Supine & Standing \\
\hline 1 & $0.14^{*}$ & 0.04 & $0.48^{*}$ & 0.02 \\
2 & 0.03 & $-0.63^{*}$ & $0.28^{*}$ & $-0.36^{*}$ \\
3 & $0.65^{*}$ & $0.21^{*}$ & $0.47^{*}$ & $0.47^{*}$ \\
4 & $0.24^{*}$ & 0.02 & 0.06 & $-0.54^{*}$ \\
5 & $0.39^{*}$ & $-0.41^{*}$ & $0.22^{*}$ & $-0.45^{*}$ \\
6 & $0.19^{*}$ & 0.24 & $0.22^{*}$ & 0.02 \\
7 & $0.29^{*}$ & $0.32^{*}$ & 0.04 & $-0.21^{*}$ \\
8 & $0.09^{\wedge}$ & $-0.23^{*}$ & $0.13^{*}$ & $-0.23^{*}$ \\
9 & $0.11^{*}$ & $-0.34^{\wedge}$ & $0.18^{*}$ & $-0.20^{*}$ \\
10 & $0.32^{*}$ & $-0.35^{*}$ & 0.01 & $-0.19^{*}$ \\
11 & $0.58^{*}$ & 0.01 & $0.63^{*}$ & 0.01 \\
12 & $0.13^{*}$ & 0.03 & $0.19^{*}$ & 0.08 \\
13 & $0.39^{*}$ & $0.51^{*}$ & $0.13^{*}$ & $0.27^{*}$ \\
14 & 0.28 & $0.33^{*}$ & 0.19 & $0.22^{*}$ \\
15 & 0.37 & $0.33^{*}$ & $0.30^{*}$ & $0.19^{*}$ \\
\hline$\wedge=\mathrm{p}<0.05 ; * \mathrm{p}<0.01$ & & &
\end{tabular}


The correlation coefficients of the individual subjects are presented in Table 1 and Table 2. Figure 1 demonstrates an example of distinctly different effect of the change in body position on the T-PCA/RR relationship in two subjects

In both positions, the intra-subject variability of QRSPCA and T-PCA was significantly smaller than the intersubject variability (Table 3, Figure 2).
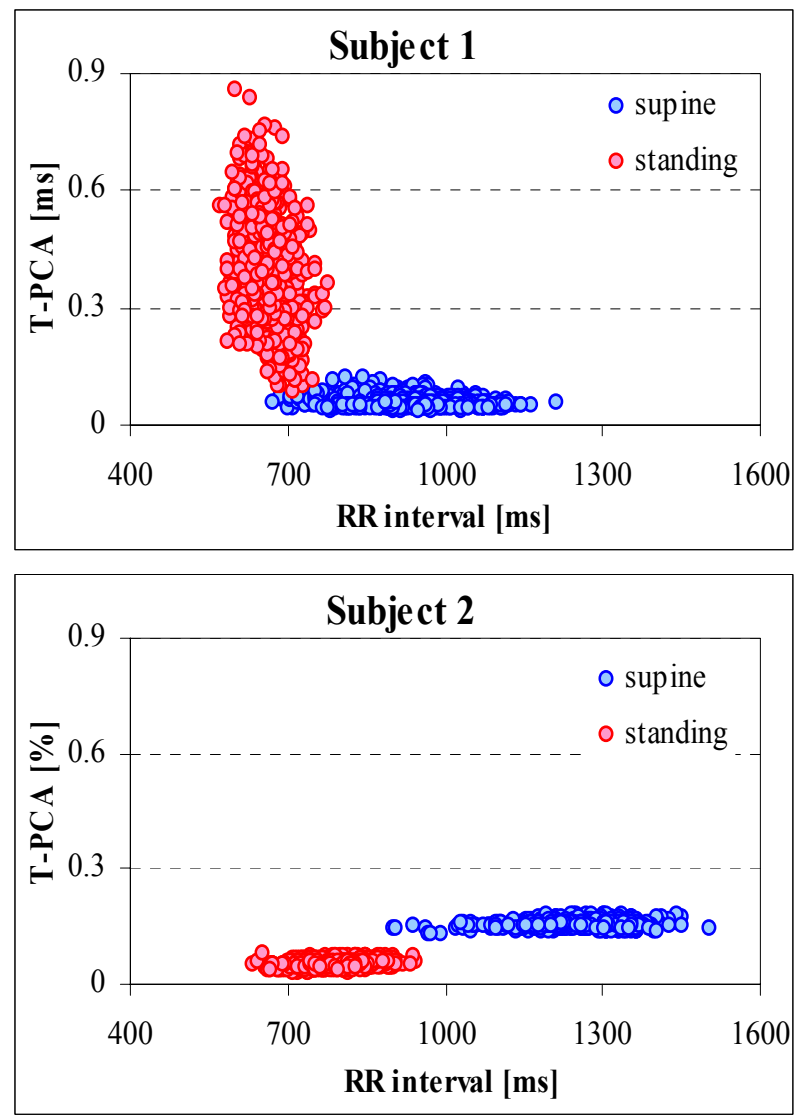

Figure 1 The figure presents an example of distinctly different change in the T-PCA/RR interval relationship with transition from supine to standing position in two subjects.

\section{Discussion and conclusions}

Our results showed that in healthy subjects investigated under relatively stable conditions the relation between QRS and T wave complexity and the RR period varies substantially between different individuals both in the supine and even more in standing position, from moderately strong negative correlation in some subjects to moderately strong positive one in others in standing position.
The reasons for this inter-individual variability are unclear, but probably include both genetic as well as environmental factors. $\mathrm{T}$ wave complexity is also influenced by the respiratory pattern (our unpublished observations), which was not controlled in this study.

The results have important clinical implications. Averaging the values of $\mathrm{T}$ wave complexity parameters across different patients and comparing various patient groups makes little sense unless the heart rate and the pattern of T-PCA/heart rate relationship in the individual patients is considered.
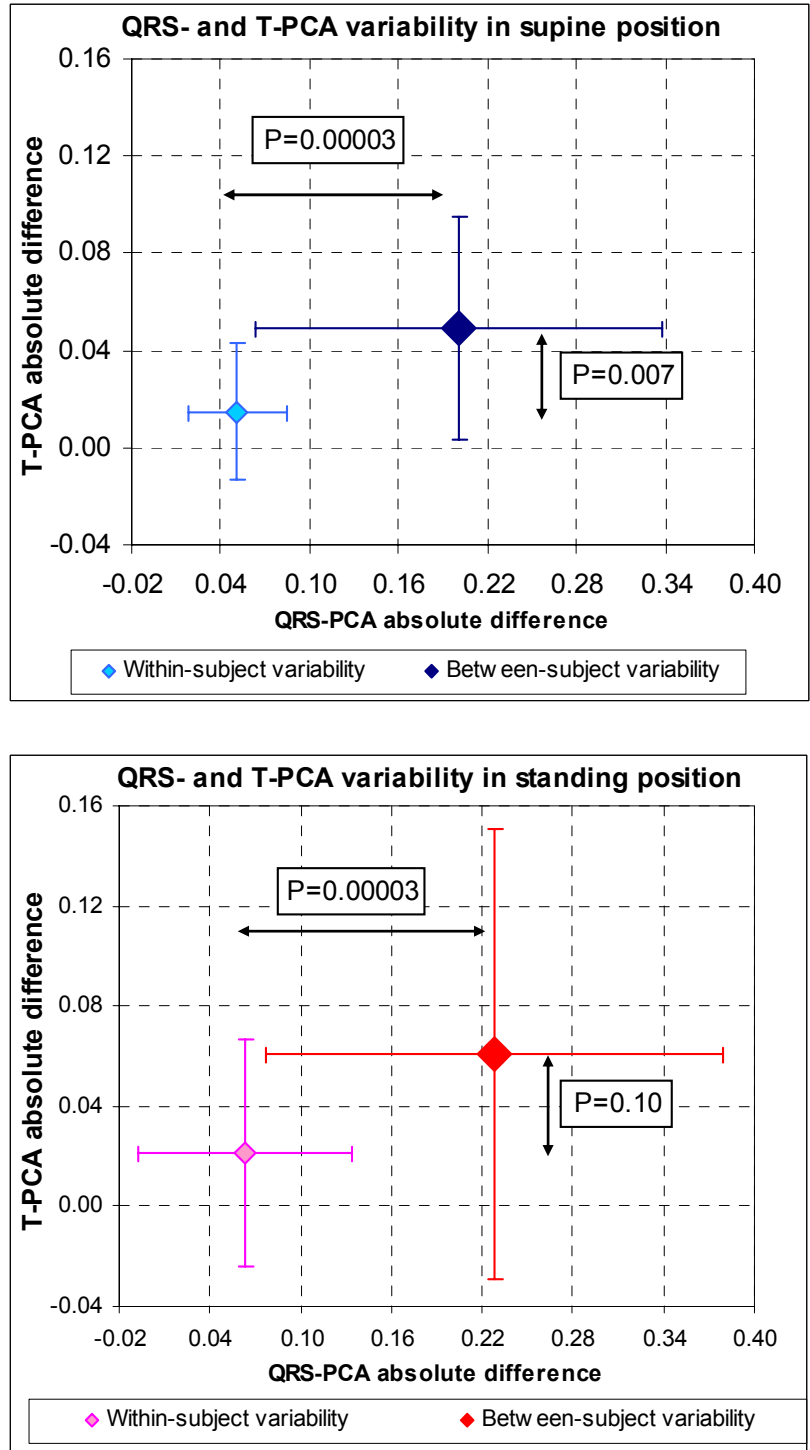

Figure 2. QRS-PCA and T-PCA variability in (a) supine position and (b) in standing position 
Table 3 Intra- and inter-subject variability of PCA-QRS and PCA-T

\begin{tabular}{cccc}
\hline \hline & $\begin{array}{c}\text { Within-subject } \\
\text { differences* }^{*}\end{array}$ & $\begin{array}{c}\text { Between-subject }_{\text {Differences^ }^{\wedge}} \\
\text { value }\end{array}$ \\
\hline Supine & & & \\
RR (ms) & $65(14,238)$ & $226(1,624)$ & 0.005 \\
PCA-QRS & $0.064(0.003$, & $0.181(0.0001$, & 0.0007 \\
$(\%)$ & $0.101)$ & $0.563)$ & \\
PCA-T (\%) & $0.006(0.0001$, & $0.027(0.0001$, & 0.0008 \\
& $0.112)$ & $0.144)$ & \\
Standing & & & $118(0,532)$ \\
RR (ms) & $66(2,165)$ & $0.211(0.004$, & 0.43 \\
PCA-QRS & $0.047(0.003$, & $0.652)$ & 0.011 \\
(\%) & $0.272)$ & $0.027(0.0004$, & 0.008 \\
PCA-T (\%) & $0.009(0.0002$, & $0.311)$ & \\
\hline \hline
\end{tabular}

$*=$ median (range) of the absolute differences between the $1^{\text {st }}$ and $2^{\text {nd }}$ recording in each subject;

$\wedge=$ median (range) of the absolute differences between the recordings of any two subjects (105 possible pairs). In each subject the average value from the two recordings has been taken.

\section{References}

[1] Priori S, Mortara D, Napolitano C, et al. Evaluation of the Spatial Aspect of the T-Wave Complexity in the Long-QT Syndrome. Circulation 1997; 96:3006-3012.

[2] Badilini F, Fayn J, Maison-Blanche P, et al. Quantitative Aspects of Ventricular Repolarization: Relationship Between Three-Dimensional T Wave loop Morphology and Scalar QT Dispersion. A.N.E. 1997; 2:146-157.

[3] De Ambroggi L, Aimè E, Ceriotti C, Rovida M, Negroni S. Mapping of Ventricular Repolarization Potentials in Patients With Arrhythmogenic Right Ventricular Dysplasia. Principal Component Analysis of the ST-T Waves. Circulation 1997; 96:4314-4318.

[4] Zabel M, Malik M, Hnatkova K, Papademetriou V, Pittaras A, Fletcher RD, Franz MR. Analysis of T-Wave Morphology From the 12-Lead Electrocardiogram for Prediction of Long-Term Prognosis in Male US Veterans. Circulation. 2002; 105:1066-1070.

[5] Okin PM, Devereux RB, Fabsitz RR, Lee ET, Galloway JM, Howard BV. Principal Component Analysis of the T Wave and Prediction of Cardiovascular Mortality in American Indians. The Strong Heart Study. Circulation 2002; 105:714

[6] Rosen, IL, Gardberg M. The effects of nonpathologic factors on the electrocardiogram. Am Heart J 1957; 53, 494-734.

[7] Karjalainen J. Functional and myocarditis-induced T-wave abnormalities. Effect of orthostasis, betablockade, and epinephrine. Chest 1983; 83, 868-874.
[8] Batchvarov VN. Circadian pattern of $\mathrm{T}$ wave morphology. In Malik M, Camm AJ (editors), Dynamic Electrocardiography, Blackwell Futura, New York 2004, 358-370.

[9] Bortolan G, Bressan M, Christov I. Gender and age influences in T-loop morphology, Comp in Card 2003;30:513-516.

[10] Bortolan G, Bressan M, Christov I. Longitudinal modifications of the T-loop morphology. Comp in Card 2002;29:685-688.

[11] Mason RE, Likar I. A new system of multiple-lead exercise electrocardiography. Am Heart J 1966; 71:196205.

[12] Christov I, Simova I. Q-onset and T-end delineation: Assessment of the performance of an automated method with the use of a reference database. Phys Meas 2007;28: 213-221.

[13] Christov II. Real time electrocardiogram QRS detection using combined adaptive threshold. Biomed Eng Online 2004;3, http://www.biomedical-engineeringonline.com $/$ content $/ 3 / 1 / 28$

[14] Daskalov IK, Dotsinsky IA and Christov II. Developments in ECG acquisition, preprocessing, parameter measurement and recording. IEEE Eng in Med \& Biol 1998;17:50-58.

Address for correspondence:

Dr. Velislav Batchvarov, MD

Division of Cardiac and Vascular Sciences,

St. George's, University of London,

Cranmer Terrace, London SW17 0RE,

United Kingdom.

e-mail: vbatchva@sgul.ac.uk 